\section{Selbstbestimmt unter Gleichen}

\author{
E. Taverna
}

Die Kontaktstelle für Selbsthilfegruppen KOSCH nennt auf ihrer "Liste 2000" über 126 gesamtschweizerisch oder überregional tätige Fachorganisationen und 300 Selbsthilfegruppen. Es gibt kaum eine Krankheit oder ein soziales Problem ohne eine Vereinigung für die Betroffenen oder deren Angehörigen. Kein Leid und kein Thema, das sich nicht teilen liesse. Wer im realen Leben keinen Anschluss findet, hat noch eine Chance im Internet, wo unzählige OnlineSelbsthilfegruppen als Forum oder Newsgroup ihre Webseiten plazieren. Das "Netz der guten Hoffnung" titelt ein NZZ-Artikel das virtuelle Angebot dieser Schicksalsgemeinschaften: "Auf ihrer der Suche nach neuen Behandlungsmöglichkeiten und guten Ärzten, nach Sicherheit und einer virtuellen Deponie für ihre Todesängste.»

\section{Fach- und ...}

Es ist nicht leicht, sich eine Übersicht über die Vielzahl von Fachgruppen, Vereinigungen und Ligen zu verschaffen. Die Dokumentationsstelle der Schweizerischen Vereinigung Pro Infirmis führt ein Organisationendossier der wichtigsten staatlichen und privaten Adressen für die Belange von Behinderten, einschliesslich Berufsverbänden, Schulen und internationalen Anlaufstellen. Das Bulletin der BehindertenSelbsthilfe Schweiz ASKIO formuliert die Grundsätze, Ziele, Aufgaben und Tätigkeitsgebiete, wie sie praktisch für jede Organisation für Patienten zutreffen. Behinderte sollen unabhängig leben und in unserer Gesellschaft mitbestimmen können. Für ein selbstbestimmtes Leben werden namentlich finanzielle Sicherheit, soziale und berufliche Integration, Abbau von architektonischen Barrieren, Wahlmöglichkeit im Bereich Wohnen und gesicherte Mobilität genannt. Die gesellschaftliche Stellung der Betroffenen wird durch den landesweiten Zusammenschluss gestärkt und die Eigenkompetenz durch Information gefördert. $\mathrm{Zu}$ den wichtigsten Gebieten von ASKIO gehören die politische Interessenvertretung, das Erarbeiten gemeinsamer Positionen sowie Informations- und Medienarbeit. Im riesigen Interessenkomplex des Gesundheitswesens ist professionelles Lobbying unverzichtbar geworden. Es geht um die Gelder der Versicherungen und Bundesstellen, der Pharmaindustrie, der Sponsoren und Mitglieder. Es geht um Einfluss auf die parlamentarische Gesetzgebung, wie bei Bauvorschriften, IV- oder BVG-Revisionen, um Sozialpolitik, wie beim Assistenzdienst und die neu disku- tierte Subjektfinanzierung durch die IV. Es geht auch um die Verteilung von Forschungsgeldern zugunsten dieser oder jener Krankheit. Gemeinsame Sekretariate unterschiedlicher Verbände, wie das "Zentrum für Selbstbestimmtes Leben" in Bern oder "Insieme» leisten die nötige Vordenkerrolle im Kräftegerangel um Steuergelder. Sie schulen die Betroffenen und Angehörigen an Tagungen, bieten Kursprogramme für die Mitarbeiter und vernetzten die internationale Gemeinschaft Gleichgesinnter. Dieser Erfahrungsaustausch ist besonders auch für Frauen wichtig, die als Behinderte oft noch zusätzlich ausgebeutet und benachteiligt werden.

\section{Selbsthilfegruppen}

Viele dieser Gruppen werden von Fachorganisationen getragen, andere sind autonom, einige politisch aktiv. Sie alle bieten einen geschützten Raum für Menschen, die einen Weg aus der Krise suchen. Ein Verzeichnis kantonaler Kontaktstellen informiert über das riesige Angebot für Familien- und Erziehungsfragen, Krankheiten und Behinderungen, psychosoziale Traumen und Sucht. Geteiltes Leid ist halbes Leid, behauptet das Sprichwort. Es hat fast für jedes Thema Platz, ob es nun viele oder wenige betrifft. Zum Beispiel für die 6600 Stomaträger, die rund 60000 Epileptiker, die 10000 MS-Betroffenen in der Schweiz, oder für die rund 15\% der Bevölkerung, die an einem Reizdarm leiden. Für Blinde und Sehbehinderte, für Amalgamgeschädigte, Gewaltopfer, Oneline-Süchtige, Stotterer, Neurodermitiker, Silikongeschädigte, Alkoholiker oder kleine Menschen. Selbsthilfegruppen haben Konjunktur, für viele Teilnehmer ist es ein Wendepunkt im Leben, wenn sie akzeptiert und verstanden werden, das verlorene Selbstwertgefühl wiederfinden. Chronisch Kranke lernen dort mit der Krankheit umzugehen und benötigen weniger Arztkonsultationen. Die praktische Hilfe und emotionale Geborgenheit der Gruppe entlastet das öffentliche Gesundheitssystem. Vereinzelte Kantone wie Basel-Stadt und Bern honorieren dies mit Beiträgen an die Kontaktstellen. Die Krankenkassen zahlen vorläufig nichts. Das BSV subventioniert über die IV jene Selbsthilfe, die sich für Behinderte einsetzt.

Fachorganisationen und Selbsthilfegruppen beeinflussen und werden beeinflusst. Interessenkonflikte sind unvermeidlich zwischen Gesunden und Kranken, zwischen Anbietern und Kunden, aber auch zwischen Funktionären und Betroffenen. Denn auch hier wäre eine Quote fällig, die wie in den USA vorschreibt, dass mindestens 51\% der Leitungs- und Arbeitsstellen von selber Behinderten eingenommen werden müssen. Oder um aus einem Artikel von ASKIO zu zitieren: "In den reichen Ländern ist der Hauptgegner nicht Vernachlässigung, Hunger, Krankheit und Tod, sondern die Vereinnahmung und Isolation von der Gesellschaft durch die politisch und finanziell starke Industrie der Sonderbetreuungsinstitutionen." 